\title{
Expression of non-muscle type myosin heavy polypeptide 9 (MYH9) in mammalian cells
}

\author{
T. Takubo, ${ }^{*}$ S. Wakui, ${ }^{\circ}$ K. Daigo, ${ }^{\circ}$ K. Kurokata, ${ }^{\circ}$ T. Ohashi, $^{\circ}$ K. Katayama, ${ }^{\circ}$ M. Hino* \\ * Clinical Hematology and Clinical Diagnostics, Graduate School of Medicine, Osaka City University, \\ Osaka, Japan; ${ }^{\circ}$ Nitto Boseki Co., Ltd., Fukushima, Japan
}

\section{(C)2003, European Journal of Histochemistry}

Myosin is a functional protein associated with cellular movement, cell division, muscle contraction and other functions. Members of the myosin super-family are distinguished from the myosin heavy chains that play crucial roles in cellular processes. Although there are many studies of myosin heavy chains in this family, there are fewer on non-muscle myosin heavy chains than of muscle myosin heavy chains. Myosin is classified as type I (myosin I) or type II (myosin II). Myosin I, called unconventional myosin or mini-myosin, has one head, while myosin II, called conventional myosin, has two heads. We transfected myosin heavy polypeptide 9 (MYH9) into HeLa cells as a fusion protein with enhanced green fluorescent protein (EGFP) and analyzed the localization and distribution of MYH9 in parallel with those of actin and tubulin. The results indicate that MYH9 colocalizes with actin stress fibers. Since it has recently been shown by genetic analysis that autosomal dominant giant platelet syndromes are MYH9-related disorders, our development of transfected EGFP-MYH9 might be useful for predicting the associations between the function of actin polymerization, the MYH9 motor domain, and these disorders.

Correspondence: Takayuki Takubo, Clinical Hematology and Clinical Diagnostics, Graduate School of Medicine, Osaka City University, 1-4-3, Asahimachi, Abeno-ku, Osaka 5458585, Japan. Phone: international +81.6.66453882

Fax: international +81.6.66453883

E-mail: m3542254@med.osaka-cu.ac.jp

Key words: green fluorescent protein, myosin heavy polypeptide 9, HeLa cells, actin, tubulin.

Paper accepted on July 14, 2003

European Journal of Histochemistry

2003; vol. 47 issue 4 [0ct-Dec]: 345-352
$\mathrm{M}$ yosin is an actin-associated protein. Through its interaction with actin, myosin induces many cell movements, including muscle contraction, cell division, axoplasmic organelle motions, as well as material transport in cells (Sanders et al. 1999; van Leeuwen et al. 1999; Mermall et al., 1998; Moores et al., 1996; DeGiorgis et al., 2002). Myosin ATPase activity produces mechanical energy derived from the hydrolysis of ATP, and myosin has many functions that use the chemical energy. Recently, gene-associated projects, such as the genome project, have made significant progress in studies of new myosin genes (Pollard et al., 2001; Win et al., 2001; Berg et al., 2001). As a result, it is known that new myosin belongs to the myosin superfamily (Berg et al., 2001). Generally, myosin is classified into muscle and non-muscle types. Muscle myosin has long been studied, but non-muscle has not. Only recent studies show that many kinds of non-muscle myosins act in cells. We therefore investigated the localization of non-muscle myosin, called myosin heavy polypeptide 9 (MYH9), in cells. MYH9, is present in many tissues including platelets. Several mutations of the MYH9 gene result in rare giant platelet disorders such as the Fechtner syndrome (FTNS), the May-Hegglin anomaly (MHA), and the Sebastian syndrome (SBS), which are autosomal dominant, and macrothrombocytopenic with characteristic leukocyte inclusions (Döhle-like bodies) (Kelley et al., 2000; Heath et al., 2001; Seri et al., 2000; Pecci et al., 2002). In these giant platelet syndromes, the Döhle-like bodies in polymorphonuclear leukocytes might be aggregates of MYH9 in the cytoplasm (Pecci et al., 2002). MYH9 is thus associated with these syndromes. Since the MYH9 gene is too large to be determined with one-step long PCR (polymerase chain reaction) methods, we obtained the full-length MYH9 gene by two-step 
methods. We performed long PCR that separated MYH9 into two fragments, and then ligated these two PCR fragments in expression vectors. To determine the MYH9 localization in cells, we used EGFP (enhanced green fluorescent protein) fusion protein methods. MYH9 has its motor domain in the N-terminal. This domain is highly conserved in all myosin heavy chains, and consists of ATPase and actinbinding sites. In order not to lose this function, we fused an EGFP tag into the C-terminal of MYH9. After the cloning of the jellyfish Aequorea victoria's cDNA (Prasher et al., 1992), many studies used GFP as a tag to demonstrate the localization of a target protein (Chalfie et al., 1994; Gerdes et al., 1996; Prasher et al., 1995; Zang et al., 1998). Because GFP is a large protein, it has some problems as a tag, but advantages as well. In order to use fluorescent GFP in transfectants, the tag detection step can be omitted; for example, by using antibody against the tag step. Thus, cells that are were still more active can be observed.

In this study, we used EGFP fusion protein with MYH9 on its C-terminal. EGFP is a variant of wildtype GFP, and has been optimized for brighter fluorescence and higher expression in mammalian cells. The EGFP gene contains more than 190 silent base changes which correspond to human-usage preferences (Haas et al., 1996), and sequences flanking EGFP have been converted to a Kozak consensus translation initial site (Kozak et al., 1987) to further increase translation efficiency in eukaryotic cells. We were interested in the relationship between MYH9 and actin stress fibers or microtubules. So, based on the demonstrated association between MYH9 and stress fibers, we induced transient overexpression of MYH9-EGFP in HeLa cells with or without treatment with cytochalasin $\mathrm{D}$. This reagent is known to profoundly disorganize the actin system (Cooper, 1987; Flanagan et al., 1980).

\section{Materials and Methods}

\section{Biochemical reagents}

Restriction enzymes (Takara and Toyobo); ligation kit and LA-PCR kit (Takara); Alpha Eagle's Modified Minimum Essential Medium (alpha MEM) (Irvine Scientific); fetal bovine serum (FBS, Invitrogen), cellfectin and OPTI-MEM (Gibco Brl); and Alexa labeled antibodies (Molecular Probes) were purchased for this study.

\section{Plasmid construction}

To make the expression vector to express MYH9 in the mammalian cells as EGFP fusion protein with C-terminal of MYH9, we separated MYH9 into two fragments for amplification by PCR. A first DNA fragment from MYH9 (25-3066), Myol, was amplified by PCR methods using HepG2 cell cDNA as template, and oligonucleotide primers myosin is (5'-GCTCTAGACATATGGCACAGC AAGCTGCCGATAAG-3') and myosin $1 \mathrm{a}$ (5'CAGCTGCTCCCGGAAGGAATTCTTCTCGT-3'), in which the underlined base sequence indicates a restriction site created for subcloning. This PCR product was digested with $\mathrm{XbaI}$ and EcoRI, and subcloned into multiple cloning sites (XbaI and EcoRI) of pUC119. The resulting plasmid vector was designated pUC119-Myol.

A second DNA fragment MYH9 (2746-5875), Myo2, was amplified by PCR methods using HepG2 cell cDNA as template and oligonucleotide primers myosin 2s (5'-CCTGACCGCCAAGAAGCAGGA ATTAGA-3') and myosin 2a (5'-GCCGGATCCGACGTCGACT T T T TCGGCAGGTTTGGCCTCAGC-3'), in which the underlined sequence indicates a restriction site created for subcloning. In myosin $2 \mathrm{a}$ primer, boldface of TTT sequences was exchanged from complementary stop codon, ATT. This fragment was subcloned into multiple cloning sites (EcoRI and BamHI) of mammalian expression plasmid vector pEGFP-N3 (Clontech) to generate pEGFP-N3-Myo2; to this aim, pUC118-Myol was digested with EcoRI and Hind III, and ligated into EcoRI and Hind III sites of pEGFP-N3-Myo2. The resulting plasmid vector, designated pEGFP-N3-MYH9 contained fulllength MYH9. This construct was confirmed by sequencing.

\section{Cell culture and transfection}

The human cell line HeLa was obtained from American Type Culture Collection (ATCC). HeLa is the first aneuploid, epithelial-like cell line to be derived from human tissue and has been maintained continuously by serial cell culture from a carcinoma of the cervix of a 31-year-old woman since 1951.

HeLa cells were cultured in alpha MEM containing $10 \%$ FBS. Media routinely included $9.6 \mathrm{mg} / \mathrm{L}$ kanamycin (Wako), $100 \mathrm{mg} / \mathrm{mL}$ streptomycin (Wako), 100kU/L penicillin (Sigma), and 9.6 $\mathrm{mg} / \mathrm{mL}$ amphotericin B (BMS). All cultures were 
incubated at $37^{\circ} \mathrm{C}$ in $6 \% \mathrm{CO}_{2}$. HeLa cells were cultured in growth media to $60 \%$ confluence. The cells were transfected using cellfectin reagent with an equal amount of pEGFP-N3-MYH9, or pEGFPN3 plasmid DNA in transfection media (antibioticfree and serum-free Opti-MEM), according to the manufacturer's instructions. Five hours after transfection, the transfected cells were washed with phosphate-buffered saline (PBS), and cultured in growth media for 48 hours. During this time, cytochalasin D (Sigma; $0.4 \mu \mathrm{g} / \mathrm{mL}$ ) or PBS (negative control) was added to the culture medium, and the cells were incubated with or without the drug for another five hours.

\section{Immunoblot analysis}

Total transfected cells without cytochalasin D on $60 \mathrm{~mm}$ dish were scraped by $0.5 \mathrm{~mL}$ SDS lysis buffer ( 50 mM Tris- $\mathrm{HCl}$ ( $\mathrm{pH}$ 8.0), 20\% SDS, 10\% glycerol, $0.0025 \%$ bromphenol blue). Whole SDS lysates were prepared by heating for $5 \mathrm{~min}$ at $95^{\circ} \mathrm{C}$ and contained 5\% 2-mercaptoethanol. SDS-polyacrylamide gel electrophoresis (SDS-PAGE) was performed on $10 \%$ gels under reducing conditions. For immunoblotting, proteins separated by SDSPAGE were transferred onto nitrocellulose membranes (Millipore pore size $0.45 \mu$ ). Transferring condition was constant current at 4 degrees, 180 minutes in transfer buffer $(20 \mathrm{mM}$ Tris, $150 \mathrm{mM}$ glycine, 20\% methanol). The molecular marker (Bio-Rad; 200 kDa (rabbit skeletal muscle myosin), 11.6KDa ( $E$. coli $\beta$-galactosidase), 97.4 $\mathrm{KDa}$ (rabbit muscle phosphorylase B), 66.2 KDa (bovine serum albumin), $45 \mathrm{KDa}$ (hen egg white ovalbumin), $32 \mathrm{KDa}$ (bovine carbonic anhydrase), $21.5 \mathrm{KDa}$ (soybean trypsin inhibitor), $14.4 \mathrm{KDa}$ (hen egg white lysozyme), 6.5 KDa (bovine pancreas aprotinin) lanes were stained using $0.1 \%$ amide black solution $(0.1 \%$ amide black, $10 \%$ acetic acid, 30\% methanol) after electro-blotting. The transferred membrane was blocked in blockace (Dainippon Pharmaceutical Inc.) for $60 \mathrm{~min}$ at $37^{\circ} \mathrm{C}$. GFP antibody (rabbit) (Clontech) as primary antibody was diluted to $1: 500$ in $5 \%$ blockace/PBS, and the membrane was incubated for 180 minutes at room temperature. The membrane was washed $3 x$ for 5 minutes in wash buffer $(0.05 \%$ Tween 20/PBS). Then HRP-conjugated anti-rabbit IgG antibody (Zymed Inc.) as secondary antibody was diluted 1:1000 in 5\% blockace/PBS, and the membrane was incubated for 60 minutes at room temperature. The membrane was washed $5 x$ for 5 minutes in wash buffer. Finally, the membrane was developed with the POD immunostain-kit (WaKo).

\section{Immunofluorescence studies}

Transfectants grown on coverslips were washed with PBS three times and fixed in $4 \%$ formaldehyde for $30 \mathrm{~min}$ at room temperature. Cells were permeable to $\mathrm{L}-\alpha$-lysophosphatidylcholine (Wako Inc.) and blocked with blockace for $60 \mathrm{~min}$ at $37^{\circ} \mathrm{C}$. Then the coverslips were incubated for 2 hours at room temperature with either: 1) a primary monoclonal antibody recognizing tubulin (Sigma Chem Co.) followed by incubation with Alexa350-conjugated anti-mouse IgG (Molecular Probe Inc.) or 2) Alexa568-conjugated phalloidin (Molecular Probe, Inc.), which was used to detect actin filaments (Cooper, 1987). Coverslips were mounted with Vectorshield (Vector Inc.).

\section{Imaging}

Slides were viewed under a fluorescence microscope (Leica). Green fluorescence was excited using a GFP-filter set (excitation $487 \mathrm{~nm}$, emission 524 $\mathrm{nm})$. Red fluorescence was excited using an Alexafilter set (excitation $592 \mathrm{~nm}$, emission $612 \mathrm{~nm}$ ). Blue fluorescence was excited using an Alexa-filter set (excitation $367 \mathrm{~nm}$, emission $452 \mathrm{~nm}$ ). The fluorescence excitation light was shuttered (shutter instruments), and exposure times were between 0.5 and $1.0 \mathrm{sec}$. Pictures were taken with a SPOT camera. The images are analyzed by the software supplied with the fluorescence microscope system (Leica).

\section{Results}

\section{MYH9 expression vector construction and fusion protein}

The full length open reading frame of the $\mathrm{MYH} 9$ cDNA consisted of 5883 nucleotides (31-5863) (Figure 1). Since MYH9 is a huge gene containing many GC-rich sequences, we could not amplify it by performing the PCR method once. We attempted to obtain two fragments of MYH9, Myol (25-3066) and Myo2 (2746-5875), by PCR using HepG2 cells cDNA (Figure 1). We constructed a mammalian expression plasmid vector containing full-length MYH9 by the following methods: Myol fragment digested with EcoRI and Hind III was ligated into EcoRI and Hind III sites of pEGFP-N3-Myo2. The resulting plasmid vector was designated $\mathrm{pEGFP}$ - 


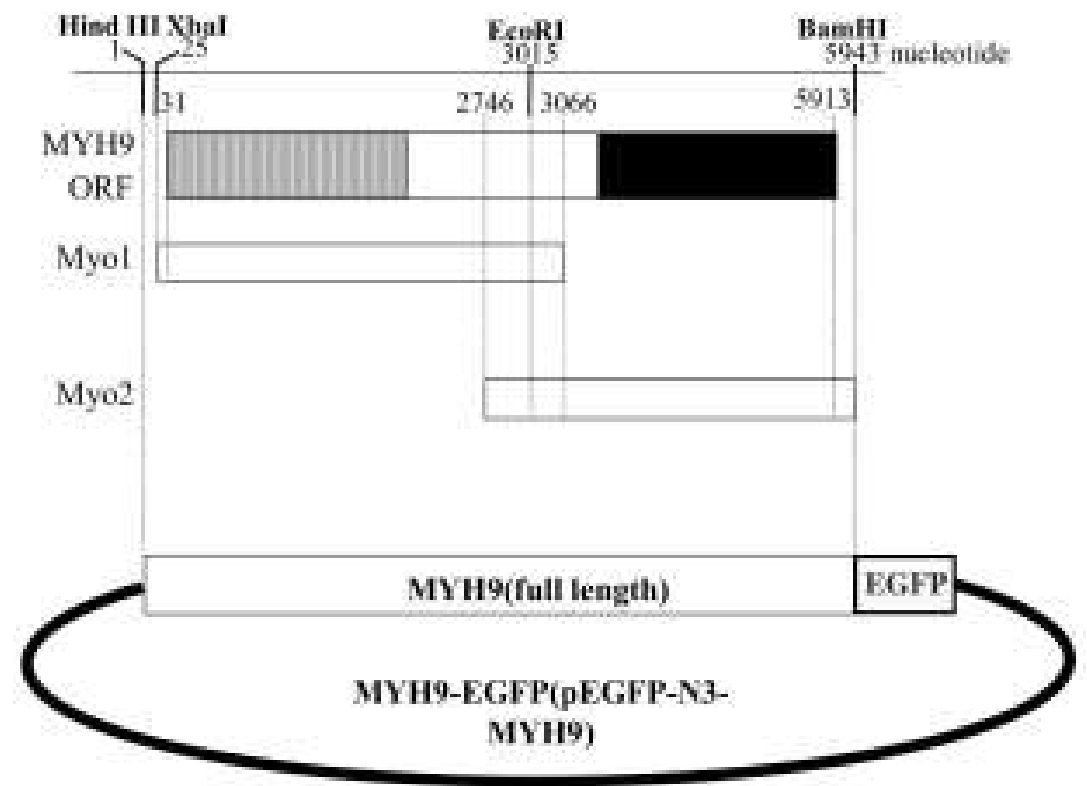

Figure 1. Schematic diagram of cDNAs for full-length non-muscle myosin heavy polypeptide 9 (MYH9). The full-length MYH9 constructs were fused to EGFP. The MYH9 domain structures in the vertically-striped box indicate the motor domain, the black box is the myosin tail domain. To make an expression vector in the mammalian cells as EGFP fusion protein with the C-terminal of MYH9, we separated MYH9 into two fragments for amplification by PCR. After the Myo1 fragment was digested with EcoRI and Hind III, and the Myo2 fragment with EcoRI and BamHI, they were inserted in pEGFP-N3. The resulting plasmid vector designated pEGFP-N3MYH9 contained full-length MYH9.

N3-MYH9 containing full-length MYH9 (Figure 1). We fused EGFP to the C-terminal of MYH9, as in Figure 1, to determine the localization of MYH9, thus allowing the motor domain of MYH9 in the Nterminus to maintain its actin-binding function.

\section{Immunoblotting analysis with MYH9-EGFP or EGFP}

HeLa cells transiently overexpressing MYH9EGFP, or only EGFP were analyzed as indicated in Figure 2. As a result of immunoblotting, a $27 \mathrm{KDa}$ band (lane 3; EGFP) was detected by anti GFP antibody in control transfectants. There was an approximately $250 \mathrm{KDa}$ band (lane 2; MYH9EGFP) obtained with the same antibody in MYH9EGFP transfectants.

\section{Localization of MYH9-EGFP in HeLa cells}

To study the cellular localization of MYH9, we transfected mammalian expression plasmid vector, pEGFP-N3-MYH9 or pEGFP-N3, into HeLa cells with cellfectin reagent. As shown in Figure 3, when only EGFP was expressed in HeLa cells, there was no regulation of its expression (Figure $3 A-E$ ). However, when MYH9-EGFP was overexpressed in HeLa cells (Figure 4), it existed with actin stress fibers (Figure 4A, B, D, E). Thus, while MYH9 colocalized with actin stress fibers, it did not colocalize with tubulin as an intermediate filament (Figure 4C, E). When cytochalasin D was added to MYH9EGFP transfectants (Figure 5), cells lost actin

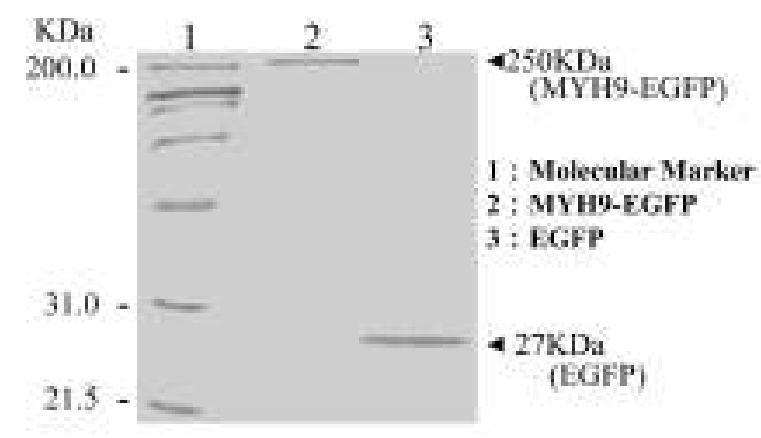

Figure 2. Immunoblotting analysis. Transiently over-expressed MYH9-EGFP or EGFP in HeLa cells. After 48 hours of transfection, the cells were dissolved with lysis buffer containing 2-mercaptoethanol. Whole-cell lysates of the cells expressing MYH9EGFP (lane 2) or EGFP (lane 3) were analyzed on $10 \%$ SDS-polyacrylamide gel in reducing conditions. After separation of proteins by SDS-PAGE, they were transferred onto nitrocellulose membranes. The molecular marker lane (lane 1) was stained with $0.1 \%$ amide black solution $(0.1 \%$ amide black, $10 \%$ acetic acid, 30\% methanol) after electro-blotting. MYH9-EGFP (250 $\mathrm{KDa}$ ) or EGFP ( $27 \mathrm{KDa}$ ) was detected by anti-GFP antibody (rabbit) as primary antibody, and HRP-conjugated anti-rabbit IgG antibody as secondary antibody.

stress fibers (Figure $5 \mathrm{~A}, \mathrm{E}, \mathrm{F}$ ). In one instance, cells lost MYH9-EGFP as well (Figure 5C-F). However, there were no changes in the localization of tubulin whether cytochalasin $D$ was added or not (Figure $4 C, E$ and Figure 5B, D, F), indicating that MYH9EGFP existed with actin, but did not affect the form of tubulin expression. 


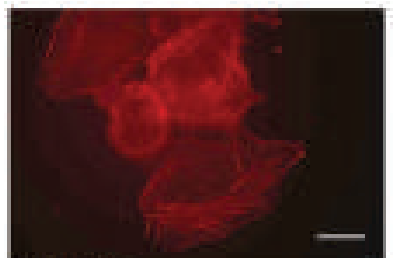

(A) actin

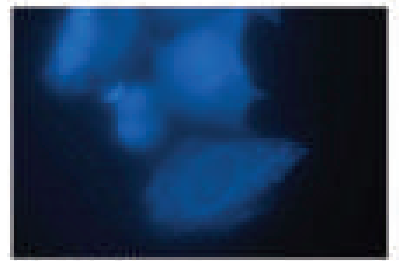

(B) tubulin

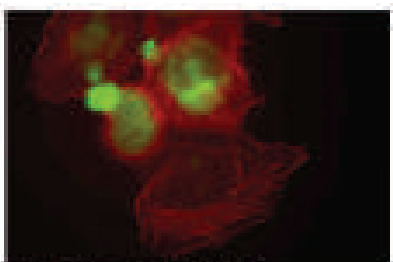

(C) actin and EGFP

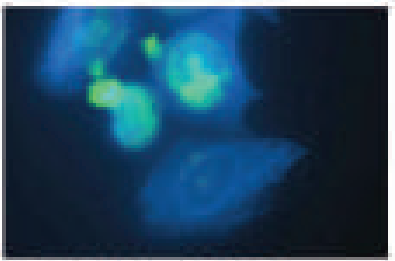

(D) tubulis and EGFT

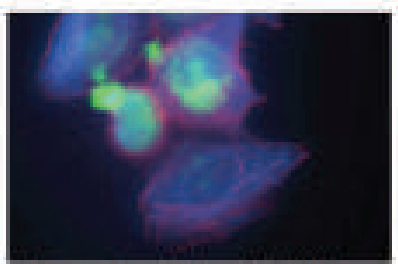

(E) octin, rubilin and EGFP

Figure 3. HeLa cells transfected with EGFP. (A) Localization of endogenous actin (red) stained with Alexa568-labeled phalloidin in EGFP transfectants. (B) Localization of endogenous tubulin (blue) stained with anti-mouse-tubulin as primary antibody and Alexa350-conjugated anti-mouse IgG as secondary antibody in EGFP transfectants. (C) Colocalization of endogenous actin (red) and EGFP (green). These did not exist together. (D) Colocalization of endogenous tubulin (blue) and EGFP (green). These did not exist together, either. (E) Colocalization of endogenous actin (red), tubulin (blue) and EGFP (green). Their expressions were independent of each other. So generally speaking, we confirmed that EGFP did not bind to cytoskeleton in the cells, i.e. to actin, tublin, etc. Bar, $10 \mu \mathrm{m}$.

\section{Discussion}

We could not observe the localization and association of MYH9 with cytoskeletal components in normal conditions (data not shown). Thus, it may be concluded that it is the overexpression of MYH9 that determines the detection of MYH9 localization and association with cytoskeletal components.

We found that EGFP, when expressed alone in HeLa cells, spread diffusely throughout the cells (Figure 3), confirming that EGFP existed independently of the cytoskeleton in these cells, i.e., independently of actin, myosin, etc. Generally speaking, using GFP as a tag is effective for studying the localization of target protein in cytoskeleton, secretory pathways, plasma membranes, nucle-

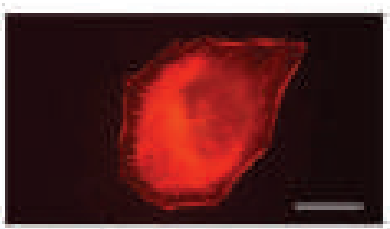

(A) actin

(B) MYH9-EGFP

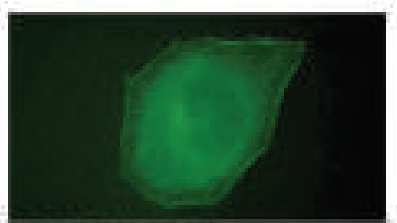

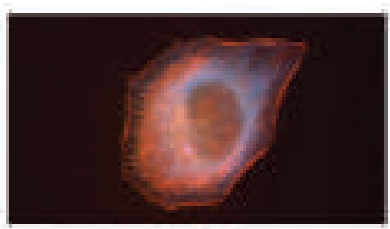

(C) actin und tubsulit

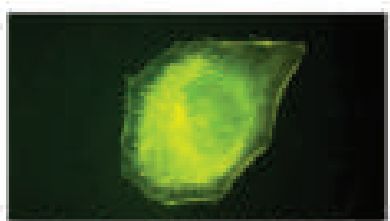

(D) actin and MYH9-EGFP

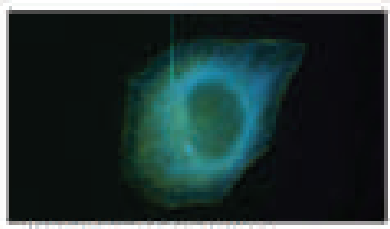

(E) actin, tabulin and MYH9-EGFP
Figure 4. HeLa cells transfected with MYH9-EGFP. (A) Localization of endogenous actin stained with Alexa568-labeled phalloidin in MYH9-EGFP transfectants (red). (B) Localization of MYH9-EGFP in HeLa of MYH9-EGFP transfectants (green). (C) Co-localization of endogenous actin (red) and tubulin (blue). These did not exist together. (D) Colocalization of endogenous actin and overexpressed MYH9-EGFP. These were expressed together in MYH9-EGFP transfectants (yellow). (E) Colocalization of endogenous actin, tubulin, and MYH9-EGFP. The localization of tubulin (blue) did not correspond to that of actin and MYH9-EGFP (yellow). The results thus indicated that while MYH9 localized with actin, it did not exist with tubulin. Bar, 10 $\mu \mathrm{m}$.

us, etc. (Gerdes et al., 1996). By comparison, in the case of EGFP, MYH9-EGFP was observed to exist at the periphery of transfectants with actin stress fibers after staining them with Alexa568 labeled phalloidin (Figure 4), indicating that MYH9-EGFP existed with $\mathrm{F}$-actin as well as with other myosins (Moores et al., 1996). However, there were no transient changes in the form of the MYH9-EGFP transfectants; i.e. stress fibers, cell adhesions, cell structures, etc. That is to say, overexpression of MYH9-EGFP in the HeLa cells did not make obvious changes in the form. We believe that MYH9 cannot have functions without actin.

When we suppressed localization of F-actin in MYH9-overexpressing cells by treatment with phalloidin, MYH9 localization was lost as well. But the 


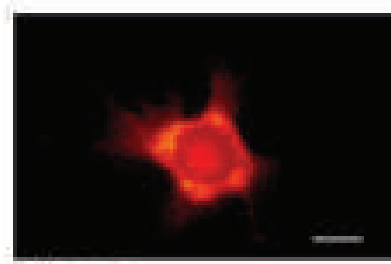

(A) acrin

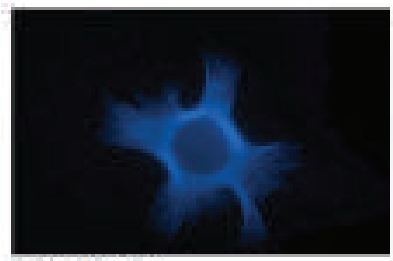

(B) tubulin

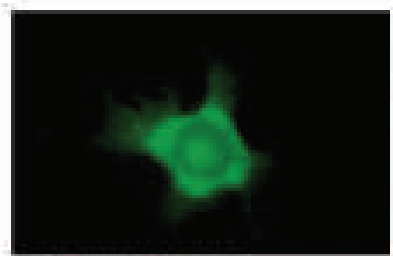

(C) MYH9-EGFP

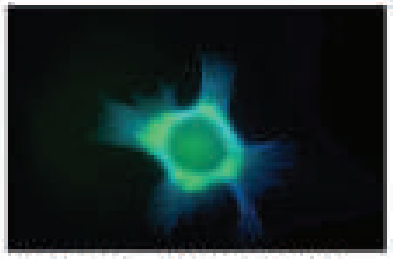

(D) tubulini MYH9-EGFP

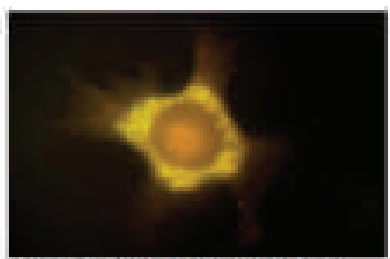

(E) actin and MYH9-EGFP

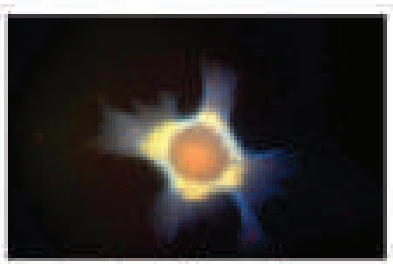

(F) actin, tubulin asd MYH9-EGFP

Figure 5. HeLa cells, treated with cytochalasin D, transfected with MYH9-EGFP. (A) Localization of endogenous actin (red) stained with Alexa568-labeled phalloidin in EGFP transfectants. Treatment with cytochalasin D disorganized the actin system.

(B) Localization of endogenous tubulin (blue). Endogenous tubulin was stained with anti-mouse-tubulin as primary antibody and Alexa-350-conjugated anti-mouse IgG as secondary antibody in MYH9-EGFP transfectants. Because we detected tubulin fibers under these conditions, there was no effect from MYH9-EGFP transfection or cytochalasin D treatment. (C) Localization of MYH9-EGFP in HeLa cells of MYH9-EGFP transfectants (green). (D) Colocalization of endogenous tubulin (blue) and MYH9-EGFP (green). Their expression sites differed. (E) Colocalization of endogenous actin (red) and MYH9-EGFP (green). These existed together, regardless of cytochalasin D treatment. (F) Colocalization of endogenous actin (red), tubulin (blue) and MYH9EGFP (green). The localization of tubulin (blue) did not correspond to that of actin and MYH9-EGFP (yellow). Thus, while MYH9 localized with actin, it did not exist with tubulin. Thus, when we used cytochalasin D in MYH9-EGFP transfectants to disorganize the actin system, MYH9, as well as actin, reacted. These results thus indicated that MYH9-EGFP existed with actin stress fiber, no matter how the actin system was disorganized by cytochalasin D. Bar, $10 \mu \mathrm{m}$.

cells did not die off, even after longer term observation (data not shown). Thus MYH9-overexpressing cells might be able to survive by switching to a resting stage in the cell cycle. The cells were able to divide normally a few times, if phalloidin was removed from the medium. These results indicate that, in the absence of actin filaments, MYH9 could not give cells their usual tension.

MYH9 has been studied in disorder-related genes

of the giant platelet disorders FTNS, MHA, and SBS. It was reported that MYH9 localization changes occur in granulocytes and platelets in these disorders (Pecci et al., 2002). Recently, some mutations of MYH9 have been found to cause these disorders (Heath et al., 2001; Seri et al. 2000; Pecci et al., 2002). Mutations of MYH9 in the motor domain were found in these patients (Heath et al., 2001; Seri et al., 2000).

We were able to observe MYH9 expression in the cleavage furrow on MYH9-EGFP transfectants but not in EGFP control transfectants (data not shown). It was reported that full length myosin II lacking this motor domain localizes to the cleavage furrow region in Dictyostelium cells (Zang et al., 1998). However, this deletion mutant of myosin II cannot bind to actin filaments (Zang et al., 1998). Thus, even if MYH9 localized to the cleavage furrow region, the cells might be abnormal in some way if they lacked this motor domain function of binding to actin filaments. Thus, one cause of these disorders might be the loss of MYH9 motor function.

Recently, it has been reported that there are more than 40 myosin genes that can be divided into 12 classes based on analysis of myosin heavy chains (Berg et al., 2001). In Dictyostelium discoideum, almost all of the myosins have been cloned. To clarify the myosin functions in Dictyostelium discoideum, myosins were knocked out one by one. Recently, as a new myosin super-family, two unique myosins were reported, MyoK (Yazu et al., 1999; Schwarz et al., 1999; Schwarz et al., 2000) and MyoM (Oishi et al., 2000; Schwarz et al., 1999). MyoK is a myosin I with the longest loop-1 insert in the head domain and the shortest tail. The insert sequences are rich in Gly, Pro, and Arg, called the GPR loop, and are presumed to be a phosphorylation sites in MyoK (Spudich et al., 1994; Schwarz et al., 2000). It is therefore supposed that there is a secondary actin-binding site in the tail. MyoK is presumed to be a cross-link of actin filament via a secondary actin-binding site, and to slide as binding actin filament strands through MIHCK (myosin I heavy chain kinase). MyoM is presumed to be myosin with a phosphorylation sensor. Following the motor domain, two calmodulin-binding IQ motifs, a putative coiled-coil region, and a Pro, Ser and Thr-rich domain combine with the dbl homology $(\mathrm{DH})$ domain, and the pleckstrin homology $(\mathrm{PH})$ domains. These are conserved in Rho GDP/GTP 
exchange factors (RhoGEFs). MyoM is suggested to play a role in Rac-mediated signal transduction and remodeling of the actin cytoskeleton. So, as MyoK or MyoM are controlled by coupling factors such as MIHCK or Rac, there might be a mechanism whereby, if only MYH9 is expressed, it does not let other factors influence the cells.

In the future, we hope to find cofactors of MYH9 using MYH9-EGFP as the tag, and we are trying to determine what is downstream. Our objective is clinical application by using this factor as a target.

\section{References}

Berg JS, Powell BC, Cheney RE. A millennial myosin census. Mol Biol Cell 2001;12:780-94.

Chalfie M, Tu Y, Euskirchen G, Ward WW, Prasher DC. Green fluorescent protein as a marker for gene expression. Science 1994; 263:802-5

Cooper JA. Effects of cytochalasin and phalloidin on actin. J Cell Biol 1987;105:1473-8.

DeGiorgis JA, Reese TS, Bearer EL. Association of a nonmuscle myosin II with axoplasmic organelles. Mol Biol Cell 2002;13:104657.

Flanagan MD, Lin S. Cytochalasins block actin filament elongation by binding to high affinity sites associated with F-actin. J Biol Chem 1980; 255:835-8.

Gerdes $\mathrm{HH}$, Kaether C. Green fluorescent protein: applications in cell biology. FEBS Lett 1996;389:44-7.

Haas J, Park EC, Seed B. Codon usage limitation in the expression of HIV-1 envelope glycoprotein. Curr Biol 1996;6:315-24.

Heath KE, Campos-Barros A, Toren A, Rozenfeld-Granot G, Carlsson $L E$, Savige J, et al. Nonmuscle myosin heavy chain IIA mutations define a spectrum of autosomal dominant macrothrombocytopenias: May-Hegglin anomaly and Fechtner, Sebastian, Epstein, and Alportlike syndromes. Am J Hum Genet 2001; 69:1033-45.

Kelley MJ, Jawien W, Ortel TL, Korczak JF. Mutation of MYH9, encoding non-muscle myosin heavy chain $\mathrm{A}$, in May-Hegglin anomaly. Nat Genet 2000;26:106-8.

Kozak M. An analysis of 5'-noncoding sequences from 699 vertebrate messenger RNAs. Nucleic Acids Res 1987;15:8125-48.

Mermall V, Post PL, Mooseker MS. Unconventional myosins in cell movement, membrane traffic, and signal transduction. Science $1998 ; 279: 527-33$
Moores SL, Sabry JH, Spudich JA. Myosin dynamics in live Dictyostelium cells. Proc Natl Acad Sci USA 1996;93:443-6.

Oishi N, Adachi H, Sutoh K. Novel Dictyostelium unconventional myosin, MyoM, has a putative RhoGEF domain. FEBS Lett 2000; 474:16-22.

Pecci A, Noris P, Invernizzi R, Savoia A, Seri M, Ghiggeri GM, et al. Immunocytochemistry for the heavy chain of the non-muscle myosin IIA as a diagnostic tool for MYH9-related disorders. Br J Haematol 2002;117:164-7.

Pollard TD. Genomics, the cytoskeleton and motility. Nature 2001; 409:842-3.

Prasher DC, Eckenrode VK, Ward WW, Prendergast FG, Cormier MJ. Primary structure of the Aequorea victoria green-fluorescent protein. Gene 1992;111:229-33.

Prasher DC. Using GFP to see the light. Trends Genet 1995;11:320-3.

Sanders LC, Matsumura F, Bokoch GM, de Lanerolle P. Inhibition of myosin light chain kinase by p2l-activated kinase. Science 1999; 283:2083-5.

Schwarz EC, Geissler H, Soldati T. A potentially exhaustive screening strategy reveals two novel divergent myosins in Dictyostelium. Cell Biochem Biophys 1999;30:413-35.

Schwarz EC, Neuhaus EM, Kistler C, Henkel AW, Soldati T. Dictyostelium myosin IK is involved in the maintenance of cortical tension and affects motility and phagocytosis. J Cell Sci 2000; 113: 621-33.

Seri M, Cusano R, Gangarossa S, Caridi G, Bordo D, Lo Nigro C, et al. Mutations in MYH9 result in the May-Hegglin anomaly, and Fechtner' and Sebastian' syndromes. The May-Hegglin/Fechtner Syndrome Consortium. Nat Genet 2000;26:103-5.

Spudich JA. How molecular motors work. Nature 1994;372:515-8.

van Leeuwen FN, van Delft $\mathrm{S}$, Kain $\mathrm{HE}$, van der Kammen RA, Collard $J G$. Rac regulates phosphorylation of the myosin-II heavy chain, actinomyosin disassembly and cell spreading. Nat Cell Biol 1999; $1: 242-8$.

Win TZ, Gachet $Y$, Mulvihill DP, May KM, Hyams JS. Two type V myosins with non-overlapping functions in the fission yeast Schizosaccharomyces pombe: My052 is concerned with growth polarity and cytokinesis, Myo51 is a component of the cytokinetic actin ring. J Cell Sci 2001;114:69-79.

Yazu M, Adachi H, Sutoh K. Novel Dictyostelium unconventional myosin MyoK is a class I myosin with the longest loop-1 insert and the shortest tail. Biochem Biophys Res Commun 1999;255:711-6.

Zang JH, Spudich JA. Myosin II localization during cytokinesis occurs by a mechanism that does not require its motor domain. Proc Natl Acad Sci USA 1998;95:13652-7. 
T. Takubo et al. 